\title{
A GENERALIZATION OF THE HAHN-MAZURKIEWICZ THEOREM
}

\author{
L. E. WARD, JR.
}

\begin{abstract}
It is proved that if a Hausdorff continuum $X$ can be approximated by finite trees (see the text for definition) then there exists a (generalized) arc $L$ and a continuous surjection $\varphi: L \rightarrow X$.
\end{abstract}

1. Introduction. The celebrated Hahn-Mazurkiewicz theorem, first proved about 1914 [4], [8], asserts that a Peano continuum is the image of $[0,1]$ under some continuous mapping. Subsequent attempts to generalize the theorem to the nonmetric setting proved unavailing, and in 1960 Mardešić [6] described a locally connected Hausdorff continuum which is not arcwise connected (in the generalized sense) and hence is not the continuous image of any arc. Later Cornette and Lehman [3] exhibited a simpler example with the same properties. The possibility remained that an arcwise connected, locally connected continuum is the continuous image of some arc, but in [7] Mardešic and Papic showed that any product of continua which is the continuous image of an arc is necessarily metrizable. Consequently, even such a nice continuum as $L \times[0,1]$, where $L$ is the "long arc", is not the continuous image of an arc. Later results of Treybig [12], [13], A. J. Ward [15] and Young [19] elaborated on this theme.

Quite recently some affirmative results have appeared. Cornette [2] proved that a tree is the continuous image of some arc, and the author [17] has extended this to rim-finite continua. Different proofs of these results have been found independently by Pearson [10], [11].

In this paper we prove a generalization of the Hahn-Mazurkiewicz theorem which includes all of the aforementioned affirmative results.

We recall some terminology. A continuum is a compact, connected Hausdorff space. An arc is a continuum with exactly two noncutpoints. A tree is a continuum in which each pair of distinct points can be separated by some point. A finite tree is a tree with only finitely many endpoints.

A continuum $X$ can be approximated by finite trees if there exists a family $\mathfrak{T}$ of finite trees such that

(1) $\mathcal{T}$ is directed by inclusion,

(2) $\cup \mathcal{T}$ is dense in $X$,

(3) if $\mathcal{Q}$ is an open cover of $X$ then there exists $T(\mathcal{Q}) \in \mathcal{T}$ such that if

Received by the editors June 6, 1975.

AMS (MOS) subject classifications (1970). Primary 54C05, 54F25; Secondary 54B25, 54D05.

Key words and phrases. Hahn-Mazurkiewicz theorem, continuum, arc, continuous image of an arc, approximation by finite trees, approximation by a sequence of finite dendrites, inverse limit. 
$T(\mathscr{U}) \subset T \in \mathcal{T}$, and if $C$ is a component of $T-T(\mathcal{U})$, then there exists $U \in \mathcal{Q}$ such that $C \subset U$.

Our principal result is the following.

THEOREM 1. If $X$ is a continuum which can be approximated by finite trees then there exists an arc $L$ and a continuous surjection $\varphi: L \rightarrow X$.

\section{Proof of Theorem 1.}

LEMMA 1. If $\left\{T_{\alpha}, r_{\beta \alpha}\right\}$ is an inverse system of trees and if the bonding mappings $r_{\beta \alpha}$ are monotone, then $T_{\infty}=\operatorname{inv} \lim \left\{T_{\alpha}, r_{\beta \alpha}\right\}$ is a tree.

Proof. Nadler [9, Theorem 3] has shown that $T_{\infty}$ is hereditarily unicoherent, and Capel [1] proved that $T_{\infty}$ is locally connected. Hence [16, Theorem 9], $T_{\infty}$ is a tree.

Lemma 2. If $T_{1}$ and $T_{2}$ are trees with $T_{1} \subset T_{2}$, then there exists a retraction $r: T_{2} \rightarrow T_{1}$ which is monotone. Moreover, if $C$ is a component of $T_{2}-T_{1}$ then $C$ has one-point boundary $x(C)$ and $r(C)=x(C)$.

Proof. If $C$ is a component of $T_{2}-T_{1}$ then, by the hereditary unicoherence of trees, $\bar{C} \cap T_{1}$ is connected. Suppose $\bar{C} \cap T_{1}$ contains distinct elements $x$ and $y$; then there are connected neighborhoods $U_{x}$ and $U_{y}$ of $x$ and $y$, respectively, such that $\bar{U}_{x}$ and $\bar{U}_{y}$ are disjoint. Since $C$ is an open set, we can invoke a standard chaining argument to show the existence of a continuum $K$ which is contained in $C$ and which meets both $U_{x}$ and $U_{y}$. If we define $P=\bar{U}_{x} \cup K \cup \bar{U}_{y}$ and $Q=\bar{C} \cap T_{1}$, then $P$ and $Q$ are subcontinua of $T_{2}, P \cap Q \subset\left(\bar{U}_{x} \cup \bar{U}_{y}\right)$, and $P \cap Q$ meets both $\bar{U}_{x}$ and $\bar{U}_{y}$. This contradicts the hereditary unicoherence of the tree $T_{2}$, and hence $\bar{C} \cap T_{1}=\bar{C}-$ $C$ consists of a single point, $x(C)$. Define $r: T_{2} \rightarrow T_{1}$ by $r \mid T_{1}=1$ and $r(C)=x(C)$ for each component $C$ of $T_{2}-T_{1}$. It is straightforward to verify that $r$ is continuous. Finally, $r$ is monotone because, for each $x \in T_{2}$,

$$
r^{-1}(x)=\{x\} \cup \cup\left\{C: C \text { is a component of } T_{2}-T_{1} \text { and } \bar{C} \cap T_{1}=\{x\}\right\} \text {, }
$$

which is a connected set.

For the remainder of this section let $X$ be a continuum which is approximated by the family $\mathcal{T}$ of finite trees. Then the system $\mathcal{T}=\left\{T_{\alpha}, r_{\beta \alpha}\right\}$ is an inverse system with monotone bonding maps, and hence $T_{\infty}=\operatorname{inv} \lim \mathcal{T}$ is a tree.

Lemma 3. If $\left(x_{\alpha}\right) \in T_{\infty}$ then $\left(x_{\alpha}\right)$ is a convergent net in $X$.

Proof. Let $p$ be a cluster point of the net $\left(x_{\alpha}\right)$ and suppose $V$ is an open set containing $p$. There exists a finite open cover $\beta$ of $X$ such that if $p \in U \in \beta$ then $\operatorname{Star}(U, \beta) \subset V$. By hypothesis there exists $T_{\beta} \in \mathcal{T}$ such that if $T_{\beta}$ $\subset T_{\gamma} \in \mathcal{T}$ and if $C$ is a component of $T_{\gamma}-T_{\beta}$, then $C$ lies in some member of $\beta$; moreover, we may assume $x_{\beta} \in U$. If $x_{\beta} \neq x_{\gamma}$ then, since $r_{\gamma \beta}\left(x_{\gamma}\right)=x_{\beta}$, it follows that the component $C$ of $T_{\gamma}-T_{\beta}$ which contains $x_{\gamma}$ has $\left\{x_{\beta}\right\}$ for boundary and hence $C \subset \operatorname{Star}(U, \beta) \subset V$. Therefore the net $\left(x_{\alpha}\right)$ converges to $p$. 
Lemma 4. The function $g: T_{\infty} \rightarrow X$ defined by $g\left(\left(x_{\alpha}\right)\right)=\lim \left(x_{\alpha}\right)$ is a continuous surjection.

Proof. Let $p=\lim \left(x_{\alpha}\right)$ and suppose $V$ is an open set containing $p$. Choose a finite open cover $\beta$ of $X$ and $T_{\beta} \in \mathcal{T}$ as in Lemma 3. If $p \in U \in \beta$, let $W=\pi_{\beta}^{-1}\left(U \cap T_{\beta}\right) \cap T_{\infty}$, a neighborhood of $\left(x_{\alpha}\right)$ in $T_{\infty}\left(\pi_{\beta}\right.$ denotes the projection function). If $\left(y_{\alpha}\right) \in W$ then $y_{\beta} \in U$ and hence, if $T_{\beta} \subset T_{\gamma} \in \mathcal{T}$, it follows that $y_{\gamma} \in \operatorname{Star}(U, \beta) \subset V$. Therefore $g\left(\left(y_{\alpha}\right)\right) \in \bar{V}$ and so $g$ is continuous.

To see that $g$ is surjective let $\left(x_{\alpha}\right) \in T_{\infty}$ with $\left(x_{\alpha}\right)$ eventually constant. That is, there exists $T_{\beta} \in \mathcal{T}$ such that $x_{\gamma}=x_{\beta}$ for all $T_{\gamma} \in \mathcal{T}$ with $T_{\beta} \subset T_{\gamma}$. Then $g\left(\left(x_{\alpha}\right)\right)=x_{\beta}$ and hence $g\left(T_{\infty}\right) \supset \cup \mathcal{T}$. Since $g$ is continuous and $\cup \mathcal{T}$ is dense in $X$ it follows that $g\left(T_{\infty}\right)=X$.

Proof of Theorem 1. By [2] and Lemma 1 there is an arc $L$ and a continuous surjection $f: L \rightarrow T_{\infty}$. By Lemma 4 the function $\varphi=g f: L \rightarrow X$ is the desired mapping.

Recently E. D. Tymchatyn [14] has applied Theorem 1 to prove that each finitely Suslinian Hausdorff continuum is the continuous image of an arc. This generalizes the result of Cornette, Pearson and the author [2], [10], [11], [17] for trees and rim-finite continua.

It is irresistible to inquire whether the condition of being approximated by finite trees is necessary as well as sufficient for a continuum to be the continuous image of an arc. I conjecture that the answer is affirmative.

3. The classical Hahn-Mazurkiewicz theorem. Recall that a dendrite is a metrizable tree. In attempting to deduce the classical theorem from Theorem 1 , we consider a metric continuum $M$. We wish to show that if $M$ can be approximated by a sequence of finite dendrites then $M$ is the continuous image of $[0,1]$. It follows from Theorem 1 that $M$ is the image of some arc, but we have no assurance that the arc is separable. The proof that $M$ is the continuous image of $[0,1]$ is facilitated by the following two lemmas.

Lemma 5. If $D$ is a finite dendrite then there exists a continuous surjection $f$ : $[0,1] \rightarrow D$.

Proof. Since $D$ has only a finite set $\left\{e_{1}, \ldots, e_{n}\right\}$ of endpoints, $n \geqslant 2$, we may write $D=A_{2} \cup \cdots \cup A_{n}$ where $A_{2}=\left[e_{1}, e_{2}\right]$ is an arc and $A_{k}=\left[d_{k}\right.$, $\left.e_{k}\right]$ is an arc irreducible between $\left(A_{1} \cup \cdots \cup A_{k-1}\right)$ and $e_{k}$ where $2<k$ $\leqslant n$. There is a homeomorphism $f_{2}:[0,1] \rightarrow A_{2}$; suppose $f_{k-1}:[0,1]$ $\rightarrow\left(A_{1} \cup \cdots \cup A_{k-1}\right)$ is a continuous surjection with $f_{k-1}(t)=d_{k}$. Without loss of generality we may assume $0<t<1$. Define

$$
\begin{gathered}
h_{1}:[0, t] \rightarrow\left[0, \frac{1}{4}\right] \text { by } h_{1}(x)=x / 4 t, \\
h_{2}:[t, 1] \rightarrow\left[\frac{3}{4}, 1\right] \text { by } h_{2}(x)=(x+3-4 t) / 4(1-t) .
\end{gathered}
$$

Let

$$
g_{1}:\left[\frac{1}{4}, \frac{1}{2}\right] \rightarrow\left[d_{k}, e_{k}\right] \text { and } g_{2}:\left[\frac{1}{2}, \frac{3}{4}\right] \rightarrow\left[e_{k}, d_{k}\right]
$$

be homeomorphisms which preserve the indicated endpoints. If we define 


$$
f_{k}= \begin{cases}f_{k-1} h_{1}^{-1} & \text { on }\left[0, \frac{1}{4}\right], \\ g_{1} & \text { on }\left[\frac{1}{4}, \frac{1}{2}\right], \\ g_{2} & \text { on }\left[\frac{1}{2}, \frac{3}{4}\right] \\ f_{k-1} h_{2}^{-1} & \text { on }\left[\frac{3}{4}, 1\right]\end{cases}
$$

then $f_{k}:[0,1] \rightarrow\left(A_{1} \cup \cdots \cup A_{k}\right)$ is a continuous surjection, and the lemma follows by induction.

Lemma 6. If $D$ and $D^{\prime}$ are finite dendrites with $D \subset D^{\prime}, r: D^{\prime} \rightarrow D$ is the natural monotone retraction and $f:[0,1] \rightarrow D$ is a continuous surjection, then there exists a monotone mapping $s:[0,1] \rightarrow[0,1]$ and a continuous surjection $f^{\prime}$ : $[0,1] \rightarrow D^{\prime}$ such that $f_{s}=r f^{\prime}$.

Proof. There are only finitely many elements $x_{1}, \ldots, x_{n}$ of $D$ which are the boundaries of components of $D^{\prime}-D$. For each $i=1, \ldots, n$ let

$$
K_{i}=\left\{x_{i}\right\} \cup \cup\left\{C: C \text { is a component of } D^{\prime}-D \text { and } x_{i} \in \bar{C}\right\},
$$

and choose $t_{i} \in f^{-1}\left(x_{i}\right)$. Without loss of generality we assume $0<t_{1}<t_{2}$ $<\cdots<t_{n}<1$. Define linear homeomorphisms $h_{0}, \ldots, h_{n}$ as follows:

$$
\begin{gathered}
h_{0}:\left[0, t_{1}\right] \rightarrow[0,1 /(2 n+1)] \text { by } h_{0}(x)=x /(2 n+1) t_{1}, \\
h_{k}:\left[t_{k}, t_{k+1}\right] \rightarrow[2 k /(2 n+1),(2 k+1) /(2 n+1)] \\
\text { by } h_{k}(x)=\left(x+2 k t_{k+1}-(2 k+1) t_{k}\right) /(2 n+1)\left(t_{k+1}-t_{k}\right), \\
k=1, \ldots, n-1, \\
h_{n}:\left[t_{n}, 1\right] \rightarrow\left[\frac{2 n}{2 n+1}, 1\right] \text { by } h_{n}(x)=\frac{x+2 n-(2 n+1) t_{n}}{(2 n+1)\left(1-t_{n}\right)} .
\end{gathered}
$$

Each of the sets $K_{i}$ is a finite dendrite, so by Lemma 5 there is a continuous surjection

$$
g_{i}:[(2 i-1) /(2 n+1), 2 i /(2 n+1)] \rightarrow K_{i}, \quad i=1, \ldots, n .
$$

Define $s:[0,1] \rightarrow[0,1]$ by

$$
\begin{array}{rlrl}
s=h_{i-1}^{-1} & \text { on }[(2 i-2) /(2 n+1),(2 i-1) /(2 n+1)], & & 1 \leqslant i \leqslant n+1, \\
s(t)=t_{i} \quad \text { if } t \in[(2 i-1) /(2 n+1), 2 i /(2 n+1)], & 1 \leqslant i \leqslant n,
\end{array}
$$

and define $f^{\prime}:[0,1] \rightarrow D^{\prime}$ by

$$
f^{\prime}=\left\{\begin{aligned}
f h_{i-1}^{-1} \quad \text { on }[(2 i-2) /(2 n+1),(2 i-1) /(2 n+1)], \\
g_{i} \text { on }[(2 i-1) /(2 n+1), 2 i(2 n+1)], \quad 1 \leqslant i \leqslant n .1
\end{aligned}\right.
$$

Then it is obvious that $s$ is continuous and monotone, that $f^{\prime}$ is a continuous surjection and that $f s=r f^{\prime}$.

We say that a metric continuum $M$ can be approximated by a sequence of finite dendrites if there exists a sequence $D_{1}, D_{2}, \ldots, D_{n}, \ldots$ of finite dendrites such that 
(1) $D_{1} \subset D_{2} \subset \cdots \subset D_{n} \subset \ldots$,

(2) $\cup\left\{D_{n}: n=1,2, \ldots\right\}$ is dense in $M$,

(3) if $C$ is a component of $D_{n+1}-D_{n}$ then $\operatorname{diam}(C)<2^{-n}$.

THEOREM 2. If $M$ is a metric continuum then the following statements are equivalent:

(i) there exists a continuous surjection $\psi:[0,1] \rightarrow M$,

(ii) $M$ is a Peano continuum,

(iii) $M$ can be approximated by a sequence of finite dendrites.

Proof. It is well known that (i) $\Rightarrow$ (ii). (For example, consult [5].)

To see that (ii) $\Rightarrow$ (iii), it is a consequence of the fact that $M$ is compact and locally connected that $M$ admits a sequence $\mathscr{U}_{n}$ of finite connected open covers such that $\mathscr{Q}_{n+1}$ refines $\mathscr{U}_{n}$ and $\operatorname{diam}(U)<2^{-n}$ for each $U \in \mathscr{U}_{n}$. Independent of the Hahn-Mazurkiewicz theorem it can be shown that each member of $\mathscr{U}_{n}$ is arcwise connected. (See [18, Chapter II, $\$ 5$, under the second remark on p. 39, together with 5.3].) Therefore it is possible to construct a sequence of finite dendrites $D_{1}, D_{2}, \ldots$ such that $D_{n}$ meets each member of $\mathscr{Q}_{n}, D_{n} \subset D_{n+1}$, and each component of $D_{n+1}-D_{n}$ lies in some member of $\mathscr{Q}_{n}$.

To prove (iii) $\Rightarrow$ (i), let $M$ be approximated by the sequence $D_{1} \subset D_{2}$ $\subset \ldots$ of finite dendrites. By Lemmas 5 and 6 there are continuous surjections $f_{n}$ and continuous monotone surjections $r_{n}$ and $s_{n}$ so that the ladder

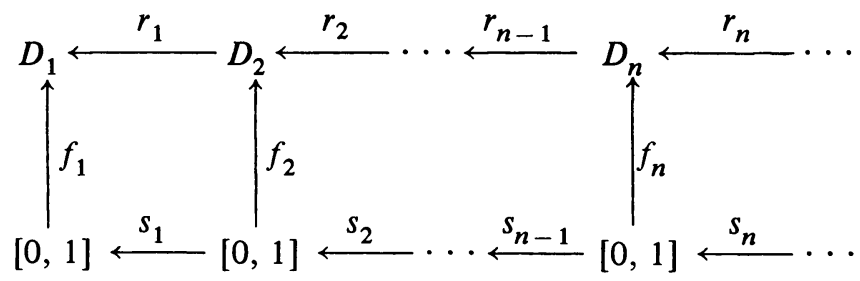

is commutative. It follows that $D_{\infty}=\operatorname{inv} \lim \left\{D_{n}, r_{n}\right\}$ is a dendrite, the limit of the inverse sequence $\left\{[0,1], s_{n}\right\}$ is $[0,1]$, and there is induced a continuous surjection $f:[0,1] \rightarrow D_{\infty}$. Lemmas 3 and 4 now apply and hence there is a continuous surjection $g: D_{\infty} \rightarrow M$. Let $\psi=g f:[0,1] \rightarrow M$.

\section{REFERENCES}

1. C. E. Capel, Inverse limit spaces, Duke Math. J. 21 (1954), 233-245. MR 15, 976.

2. J. L. Cornette, Image of a Hausdorff arc is cyclically extensible and reducible, Trans. Amer. Math. Soc. 199 (1974), 253-267.

3. J. L. Cornette and B. Lehman, Another locally connected Hausdorff continuum not connected by ordered continua, Proc. Amer. Math. Soc. 35 (1972), 281-284. MR 46 \#6320.

4. H. Hahn, Mengentheoretische Charakterisierung der stetigen Kurven, Sitzungsber. Akad. Wiss. Wien 123 (1914), 2433-2489.

5. J. G. Hocking and G. S. Young, Topology, Addison-Wesley, Reading, Mass., 1961. MR 23 \# A2857.

6. S. Mardesic, On the Hahn-Mazurkiewicz theorem in nonmetric spaces, Proc. Amer. Math. Soc. 11 (1960), 929-937. MR 22 \#464.

7. S. Mardesic and P. Papic, Continuous images of ordered continua, Glasnik Mat.-Fiz. Astronom. Drustro Mat. Fiz. Hrvatske Ser. II 15 (1960), 171-178. MR 24 \# A536. 
8. S. Mazurkiewicz, Sur les lignes de Jordan, Fund. Math. 1 (1920), 166-209.

9. S. B. Nadler, Jr., Multicoherence techniques applied to inverse limits, Trans. Amer. Math. Soc. 157 (1971), 227-234. MR 43 \#5482.

10. B. J. Pearson, Mapping an arc onto a dendritic continuum, Colloq. Math. 30 (1974), 237-243.

11. __ Mapping arcs and dendritic spaces onto netlike continua, Colloq. Math. 34 (1975), $39-48$.

12. L. B. Treybig, Concerning continuous images of compact ordered spaces, Proc. Amer. Math. Soc. 15 (1964), 866-871. MR 29 \#5218.

13. Concerning continua which are continuous images of compact ordered spaces, Duke Math. J. 32 (1965), 417-422. MR 32 \#4673.

14. E. D. Tymchatyn, The Hahn-Mazurkiewicz theorem for finitely Suslinian continua (to appear).

15. A. J. Ward, Notes on general topology. III, A non-metric image of an ordered compactum, Proc. Cambridge Philos. Soc. 61 (1965), 881-882. MR 32 \# 1685.

16. L. E. Ward, Jr., Mobs, trees and fixed points, Proc. Amer. Math. Soc. 8 (1957), 798-804. MR 20 \#3516.

17. , The Hahn-Mazurkiewicz theorem for rim-finite continua, General Topology and Appl. (to appear).

18. G. T. Whyburn, Analytic topology, Amer. Math. Soc. Colloq. Publ., vol. 28, Amer. Math. Soc., Providence, R. I., 1942. MR 4, 86.

19. G. S. Young, Jr., Representations of Banach spaces, Proc. Amer. Math. Soc. 13 (1962), 667-668. MR 26 \#574.

Department of Mathematics, University of Oregon, Eugene, Oregon 97403 